EDITORIAL

\title{
Climate change research in Sri Lanka - Are we investing enough?
}

Multi-faceted adverse impacts of climate change (CC) are one of the most critical threats faced by the global community in the $21^{\text {st }}$ century. Their implications for food security, ecosystem sustainability including biodiversity and human welfare are enormous. During the last two decades since the initial Earth Summit in Rio de Janeiro in 1992, the policymakers have grappled with the challenge of formulating and implementing effective CC mitigation strategies by trying to strike a balance between reduction of anthropogenic emission rates of atmosphere-warming greenhouse gases and the increasing energy needs for economic development in both the developed and developing world. Therefore, it has been incumbent on the scientific research community to produce reliable data on the processes, mechanisms and predictions of magnitudes and impacts of $\mathrm{CC}$, globally, regionally and locally for short, medium and long-term time scales. While there is a considerable degree of clarity and understanding on the processes and mechanisms of $\mathrm{CC}$, there is a significant degree of uncertainty in the predictions on the magnitudes and impacts of CC despite substantial progress in research. For example, recent research has shown that the capacity of the terrestrial biosphere for CC mitigation through absorption of part of the $\mathrm{CO}_{2}$ emissions to the atmosphere by using it for photosynthesis (i.e. 'the land carbon sink') could decrease significantly during the course of this century, thus leading to a positive feedback on global warming (Cox et al., 2000). This could have important implications for international agreements on increasing the land carbon sink (i.e. Kyoto Protocol, REDD).

However, there is still considerable uncertainty in the estimated magnitudes of reduction in the land carbon sink. Simulation results of 11 coupled climate-carbon cycle models (Friedlingstein et al., 2006) showed unanimous agreement on the reduction of the efficiency of carbon absorption capacity of both the land and ocean sinks, thus causing a larger fraction of the anthropogenically emitted $\mathrm{CO}_{2}$ to remain in the atmosphere. However, estimates of the resulting increases in atmospheric $\mathrm{CO}_{2}$ concentration $\left(\mathrm{C}_{\mathrm{a}}\right)$ by 2100 varied from 20 to $200 \mathrm{ppm}$ for the different models making the predicted global average $\mathrm{C}_{\mathrm{a}}$ by 2100 to be within the range between 727 and 1009 ppm, with estimates of the additional atmospheric warming ranging from 0.1 to $1.5^{\circ} \mathrm{C}$. In a re-analysis of the uncertainty of the impact of reduced land carbon sink capacity, Booth et al., (2012) predicted an even wider range for the predicted global average $\mathrm{C}_{\mathrm{a}}$ by 2100 of 669 $-1139 \mathrm{ppm}$. A majority of these models predict that the terrestrial biosphere of the earth would be a net carbon source by 2100 because increased respiratory, both autotrophic and heterotrophic, losses of $\mathrm{CO}_{2}$ in a warmer environment would outweigh the increased carbon gains by vegetation, resulting from the $\mathrm{CO}_{2}$-fertilization effect due to increased $\mathrm{C}_{\mathrm{a}}$. This would be in addition to the increased anthropogenic emissions of $\mathrm{CO}_{2}$ due to fossil fuel burning and land use change such as deforestation.

While there has been intense research activity on all aspects of CC in the global scientific landscape, there has been comparatively little research activity in Sri Lanka during the last two decades. Analysis of long-term temperature and precipitation trends in different climatic zones of Sri Lanka (De Costa, 2008) has clearly shown that the rates of warming at many locations during the past century have exceeded the global average for the same period. Similarly, a clear downward trend in precipitation was shown, especially in the central highlands, which is the major catchment for the country's river systems. The above upward trends in temperature and downward trends in precipitation have been confirmed in the predicted outputs of an intercomparison of nine dynamic global vegetation models (Sitch et al., 2012). Outputs from the global research community, as outlined in the previous paragraph, point towards a high likelihood of accelerated $\mathrm{CC}$ because of the numerous positive feedbacks in the global biogeochemical (i.e. land carbon sink) and biogeophysical (i.e. evapotranspiration and the hydrologic cycle) processes and the lack of significant progress on a global agreement to reduce anthropogenic greenhouse gas emissions. Implications of accelerated CC on Sri Lanka, which has already experienced a rate of warming, which is above the global average warming 
during the last century, would be enormous during the course of the $21^{\text {st }}$ century. Of particular concern in this regard would be the possible $\mathrm{CC}$ impacts on the tropical rainforests and montane forests in the Wet Zone of Sri Lanka, which are ecosystems of extreme importance in terms of climate change mitigation and biodiversity conservation. They are also critically important to ensure water security in Sri Lanka as several major rivers originate from them. Global circulation models coupled to dynamic vegetation and land carbon cycle models have predicted large scale forest dieback in Amazonia due to $\mathrm{CC}$-induced reductions in precipitation (Betts et al., 2004). The consequent land surface change is predicted to cause several positive feedbacks to accelerate global warming. It is important to investigate whether such phenomena are replicated in the Sri Lankan tropical rainforests as well. It is worth noting that dieback has been reported in the montane forests in the Horton Plains during the last two decades.

Hence, significant investments are needed, both globally and locally, in $\mathrm{CC}$ research leading to greater understanding of the processes and mechanisms and better predictions with less uncertainty on the magnitudes and impacts of $\mathrm{CC}$. Here, it is pertinent to question whether adequate investment in $\mathrm{CC}$ research has taken place in Sri Lanka in the recent past. It is heartening to note that $\mathrm{CC}$ is one of the priority areas identified in the National Thematic Research Programme of the National Science Foundation of Sri Lanka. It is essential that this thematic area is provided substantial and broad-based financial support to carry out $\mathrm{CC}$ research including that of fundamental nature, whose outputs may not yield 'products that are competitive in the marketplace'. It is also pertinent to note that there is no co-ordinated national strategy to combat the impending adverse impacts of $\mathrm{CC}$ on different sectors of the national economy, on different geographical zones of the country and on different strata of its population. While investment on community-based local action is no doubt important, it is equally important to realize the importance of generating valid research data and outputs, which should underpin any effective national or regional action programmes for adaptation to and mitigation of CC. Therefore, it is imperative that national investment on $\mathrm{CC}$ research is increased substantially to enable capacity building in terms of both infrastructure and trained manpower to enable $\mathrm{CC}$ research whose quality measures up to the global standards.

\section{REFERENCES}

1. Betts R.A., Cox P.M., Collins M., Harris P.P., Huntingford C. \& Jones C.D. (2004). The role of ecosystem-atmosphere interactions in simulated Amazonian precipitation decrease and forest dieback under global climate warming. Theoretical and Applied Climatology 78(1-3): 157-175.

2. Booth B.B. et al., (10 authors) (2012). High sensitivity of future global warming to land carbon cycle processes. Environmental Research Letters 7: 024002 (http://iopscience.iop.org/1748-9326/7/2/024002).

3. Cox P.M., Betts R.A., Jones C.D., Spall S.A. \& Totterdell I.J. (2000). Acceleration of global warming due to carbon cycle feedbacks in a coupled climate model. Nature $\mathbf{4 0 8}$ (6809): 184-187.

4. De Costa W.A.J.M. (2008). Climate change in Sri Lanka: myth or reality? - Evidence from long-term meteorological data. Journal of the National Science Foundation of Sri Lanka 36 (Special Issue): 83-109.

5. Friedlingstein P. et al., (25 authors) (2006). Climate-carbon cycle feedback analysis: Results from C4MIP model intercomparison. Journal of Climate 19(14): 3337-3353.

6. Sitch S. et al., (27 authors) (2012). Trends and drivers of regional sources and sinks of carbon dioxide over the past two decades. Biogeosciences (in Press).

W.A.J.M. De Costa 